\title{
Deshidratador solar híbrido para el secado de plátano
}

\section{Banana drying hybrid dehydrator}

CAMACHO-MARTINEZ, Humberto Armando*广, ANTONIO-GORDILLO, Diana Montserrat, ROBLES-OCAMPO, José Billerman y SEVILLA-CAMACHO, Perla Jazmín

Universidad Politécnica de Chiapas, Ingeniería en Energía

ID $1^{\text {er }}$ Autor: Humberto Armando, Camacho-Martinez / ORC ID: 0000-0003-4864-7586, CVU CONACYT ID: 999386

ID $1^{\text {er }}$ Coautor: Diana Montserrat, Antonio-Gordillo / ORC ID: 0000-0002-3979-0455 CVU CONACYT ID: 1019863

ID $2^{\text {do }}$ Coautor: José Billerman, Robles-Ocampo / ORC ID: 0000-0002-0094-8710, CVU CONACYT ID: 166936

ID $3^{\text {er }}$ Coautor: Perla Jazmín, Sevilla-Camacho / ORC ID: 0000-0002-9702-1929 CVU CONACYT ID: 221004

DOI: $10.35429 / J O E S .2019 .21 .6 .26 .33$

Recibido 09 Septiembre, 2019; Aceptado 28 Diciembre, 2019

\section{Resumen}

En este trabajo se presenta el diseño de un secador solar para el deshidratado de frutas. Para fines de análisis y evaluación se utiliza plátano como insumo. El sistema se abastece de la energía solar en forma fotovoltaica (PV) y fototérmica (PT). La parte PV del deshidratador consta de un panel solar, esta energía alimenta resistencias eléctricas para aumentar la temperatura del deshidratador. Asimismo, el sistema PV alimenta a un sistema de control y a un par de ventiladores, estos últimos forzan la circulación de aire dentro del sistema. Los ventiladores se localizan a la entrada y a la salida del deshidratador. Por otro lado, la parte PT se compone de un sistema de almacenamiento pasivo y captación de calor directa e indirecta. Para la elaboración del secador solar fue necesario tener en cuenta ciertos parámetros como: las condiciones meteorológicas (radiación solar, temperatura ambiente y velocidad del viento), conductividad térmica, temperatura y propiedades de los componentes.

Híbrido, Coductividad térmica, Húmedad, Convección

\begin{abstract}
In this work the design of a solar dryer for the dehydration of fruits is presented. Banana is used as raw material for analysis and evaluation purposes. The system is supplied with solar energy in the form of (photovoltaic) PV and (photothermic) PT. The PV part of the dehydrator consists of a solar panel, whose energy supplies electrical resistances to increase the temperature, also feeding a forced air convection system which is carried out with a pair of fans that are at the entrance and exit of the dehydrator, also a control and measurement system. In the other side the PT part consist in a passive storage system of heat direct and indirect catchment of radiation. For the development of the solar dryer it is necessary to take into account certain parameters such as: weather conditions (solar radiation, ambient temperature and wind speed), thermal conductivity, temperature and properties of the components.
\end{abstract}

Hybrid, Thermal Conductivity, Humidity, Convection

Citación: CAMACHO-MARTINEZ, Humberto Armando, ANTONIO-GORDILLO, Diana Montserrat, ROBLESOCAMPO, José Billerman y SEVILLA-CAMACHO, Perla Jazmín. Deshidratador solar híbrido para el secado de plátano. Revista de Sistemas Experimentales. 2019. 6-21: 26-33 


\section{Introducción}

El secado de alimentos mediante la exposición a la radiación solar se ha realizado anteriormente como método práctico y de bajo costo de operación. Hoy en día, la deshidratación de alimentos sigue vigente tanto para productos comerciales que adquieren un valor agregado, así como para comunidades que utilizan los deshidratadores como su principal medio de conservación de alimentos.

En este trabajo se presenta el diseño y caracterización de un deshidratador solar híbrido, para la localidad de Suchiapa, Chiapas. El diseño de secadores solares se lleva a cabo para el secado de frutas, verduras o semillas con un funcionamiento directo y con circulación natural. Este proceso deriva en una disminución de contenido de agua y disminución de la cantidad de microorganismos del producto. Con este diseño se obtiene beneficios economicos y productivos por parte del productor, y al usar fuentes de energía renovables se evita contaminar el medio ambiente por el uso energía convencional. El uso del deshidratador solar otorga valor agregado a los excedentes de producción, teniendo así, más tiempo para comercializar o aprovechar los alimentos.

El sistema de deshidratado esta compuesta por: un colector térmico, compuesta por un área de $0.89 \mathrm{~m}^{2}$ para la captación de radiación solar; por un sistema de almacenamiento pasivo de energía, integrada por por rocas; por un sistema de control, que se encarga de regular la temperatura del interior del deshidratador; y por resistencia eléctricas para darle estabilidad térmica durante dias pocos favorables.

La convección forzada de aire en conjunto con el calor generado por las resistencias eléctricas le otorga al deshidratador una mayor tasa de protección con respecto al tiempo. La implementación de las resistencias y los ventiladores aumentan la velocidad y eficiencia del deshidratador.

El tiempo de secado de las frutas depende de varios factores tales como: tipo de producto, tamaño de los trozos, temperatura del aire, humedad relativa del aire, velocidad del aire. Para el secado se deben de tomar en cuenta distintas etapas:
Inducción inicial: Cuando un sólido se coloca en un deshidratador, la absorción de calor y su temperatura hasta la fija de secado. Conforme la temperatura aumenta, la humedad dentro del sólido disminuye, hasta alcanzar un punto de equilibro [4].

Período de velocidad constante: En la Fig. 1 el punto "B" la temperatura se estabiliza y se comienza a crear una capa superficial de humedad. Entro los puntos "B" y "C" la evaporación desde la superficie es remplazada por la difusión del agua en el interior de sólido a la misma velocidad que lo hacía en la evaporación. Se estabiliza la velocidad de secado/unidad en la superficie [4].

Decaimiento de la velocidad: En el punto "C", el agua superficula se mantiene para conservar la humedad superficial. Comienzan a apararecer puntos en la superficie en la que la velocidad decae. Esto lleva el nombre humedad crítica [4]. Humedad crítica: El punto "D" es conocido como el segundo punto crítico, aquí deje ade ser constante la velocidad, el água superficial ya a sido evaporada. En este punto las variables más importantes pasan a ser la porosidad y el tamaño de particula del sólido en cuestión. Entre los puntos "D" y "E" la velocidad decae rapidamente $y$ se denomina como "Segundo periodo de disminución de velocidad. En el punto E la velocidad de decae por completo, llegando a 0 y comienza el solido a entrar en equilibrio con el medio [4].

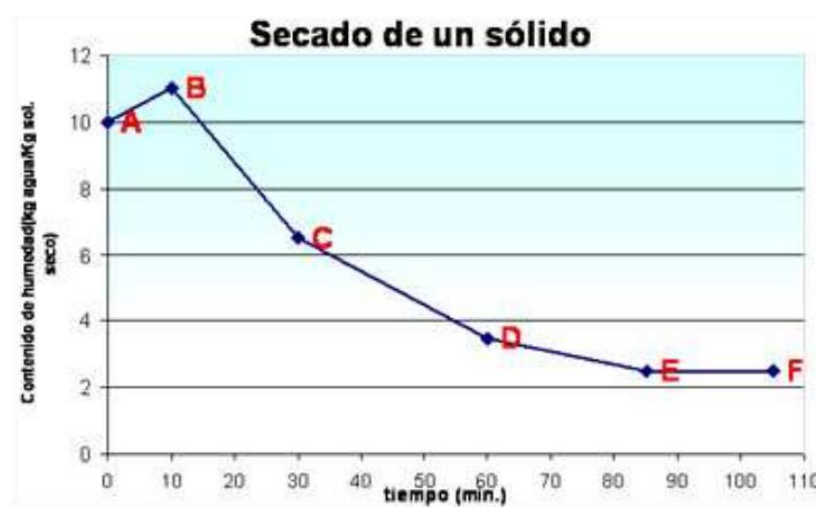

Figura 1 Perfil de secado de un sólido [5] 


\section{Metodología}

El prototipo desarollado es de carácter experimental, del cual se evalúa el desempeño y eficiencia del diseño de un deshidratador solar mixto o híbrido, que consta de una cámara de deshidratado, que permite la ejecución de procesos de transferencia de masa y calor para la obtención de alimentos deshidratados; un colector solar que en el interior cuenta con rocas el cual se encarga de absorber la energía térmica y transferirla hacia la cámara de secado, así como el aprovechamiento del efecto invernadero para aumentar la temperatura dentro de sistema; y un panel FV el cual alimenta los sistemas de control y medición como una resistencia eléctrica que tiene el fin de aumentar la temperatura del sistema.

\section{Sistema de control y medición}

El sistema de control y medición se basó en la plataforma Arduino, este consta de dos partes principales: La programación lógica y el circuito electrónico. La primera de estos dos cumple con tres diferentes propositos:

Sensar las temperaturas en diferentes puntos del deshidratador en tiempo real. Controlar la convección por medio de realys y dictamenes lógicos que tienen como variante principal la temperatura promedio de la cámara de secado.

Crear una base de datos para su posterior estudio y analsis, con las mediciones obtenidas de los diferentes sensores incluidos en el sistema, así como de promedios tanto de la cámara de secado como del colector.

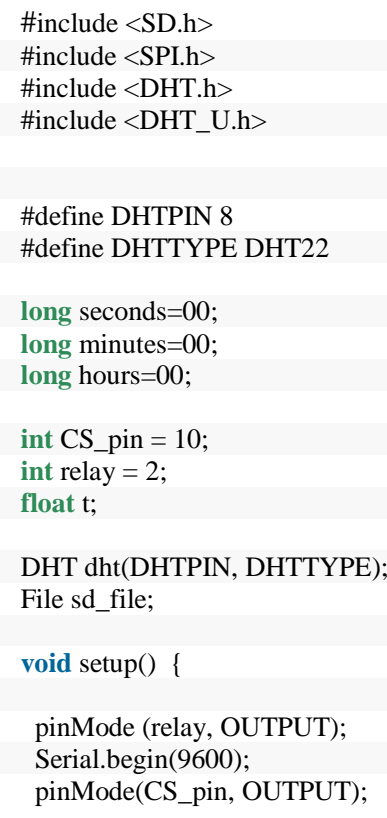

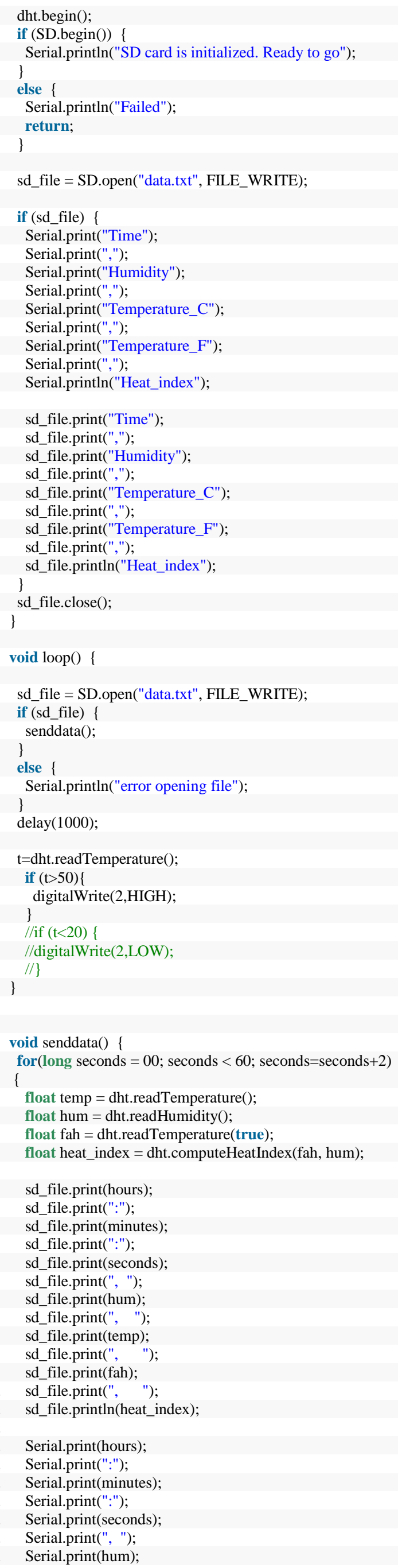

CAMACHO-MARTINEZ, Humberto Armando, ANTONIOGORDILLO, Diana Montserrat, ROBLES-OCAMPO, José Billerman y SEVILLA-CAMACHO, Perla Jazmín. Deshidratador solar híbrido para el secado de plátano. Revista de Sistemas Experimentales. 2019 


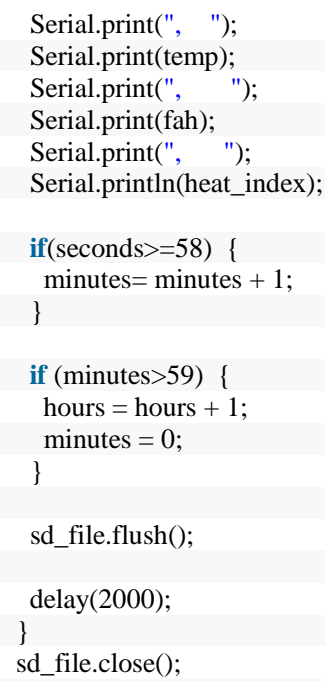

Figura 2 Código utilizado para el sistema de control y medición

El sistema consta de 5 sensores DHT 22 para las mediciones de temperatura, así como un datalogg $\operatorname{lm} 6490$,como relays convencionales, una pantalla lcd para poder obtener una lectura en tiempo real, un par de ventiladores de $12 \mathrm{~V}$, los cuales tienen la función de forzar la circulación del aire y conseguir la condición de convección forzada dentro del deshidratador.

\section{Cálculos}

\section{Aporte energetico del almacenamiento pasivo}

Se calcula la cantidad teórica que las piedras pueden ofrecer como sistema de almacenamiento pasivo, teniendo como muestra una piedra escogida al azar de las usadas.

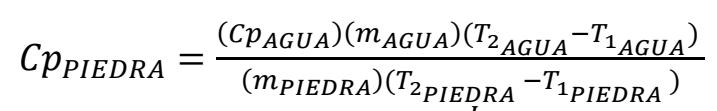

Prueba 1: $C p_{\text {PIEDRA }}=1650 \cdot \frac{\mathrm{J}}{\mathrm{kg}}{ }^{\circ} \mathrm{C}$

Prueba 2: $C p_{\text {PIEDRA }}=1691.54 \frac{\mathrm{J}}{\mathrm{kg}}{ }^{\circ} \mathrm{C}$

Prueba 3: $C p_{\text {PIEDRA }}=1772.09 \frac{\mathrm{J}}{\mathrm{kg}}{ }^{\circ} \mathrm{C}$

Promedio de $C p_{P I E D R A}=1704.63 \frac{\mathrm{Jg}}{\mathrm{kg}}^{\circ} \mathrm{C}$

La cantidad de energía que puede ser absorbida por el sistema de forma ideal es de:

$C p_{\text {total }}=2.56 \mathrm{MJ}{ }^{\circ} \mathrm{C}$

Teniendo en el porcentaje de energía que se puede recuperar de un lecho de piedras es de aproximadamente el $40 \%$ [6].

$C p_{\text {totalreal }}=1024 \mathrm{~kJ}^{\circ} \mathrm{C}$

\section{Energía aportada por la resistencia eléctrica}

Se instalo en el interior de la cámara de deshidratado una resistencia eléctrica la cual es alimentada por un panel FV de DC, el cual tiene una potencia de $90 \mathrm{~W}$. Teniendo en cuenta la literatura una resistencia eléctrica tiene una eficiencia que ronda el 58.3\% [7]. Por lo que se puede calcular la energía aportada:

$E r=90 W * 0.583 * 3600 s$

$E r=186 \mathrm{~kJ}$

\section{Energía de deshidratación}

Donde " $m_{f p}$ " es la masa de agua pérdida en el proceso $(\mathrm{kg})$; " $\mathrm{Cv}$ ", el calor latente del agua a temperatura media $(\mathrm{kJ} / \mathrm{kg})$; " $\mathrm{m}_{\mathrm{a}}$ ", la masa del aire en un instante dado; " $\mathrm{C} a$ ", el calor específico del aire a presión constante $\left(\mathrm{kJ} / \mathrm{kg}^{*} \mathrm{~K}\right)$; y temperatura de ingreso y salida del aire $\left({ }^{\circ} \mathrm{C}\right)[3]$.

$Q_{d}=m_{f p}(C v)$

$Q_{d}=1,332 \mathrm{~kJ}$

\section{Consumo específico de calor}

En la ecuación 3, donde $H_{2}$ es la entalpía final, $H_{1}$ es la entalpía inicial, $X_{2}$ es el contenido de humedad final y $X_{1}$ es el contenido de humedad inicial. Cada valor de las variables hace referencia al aire seco [1].

$$
\begin{aligned}
& q=\frac{\left(H_{2}-H_{1}\right)}{\left(X_{2}-X_{1}\right)} \\
& q=2,473.68 \frac{\mathrm{kJ}}{\mathrm{kg}}
\end{aligned}
$$

\section{Coeficiente global de perdidas}

$\mathrm{U}_{\text {sup }}$ es la conductividad térmica en la parte superior. En la ecuación 5 el calor se transfiere entre la placa de absorción y la cubierta interior por convección y radiación en forma paralela; esto mismo, también ocurre entre las dos cubiertas, interior y exterior. Los mismos mecanismos de transferencia son igualmente importantes en la disipación de calor por parte de la cubierta exterior hacia el ambiente $[1,2]$.

$U_{\text {sup }}=\left\{\frac{N}{\frac{C}{T_{p m}}\left[\frac{\left(T_{p m}-T_{a}\right)}{(N-f)}+\frac{1}{h_{w}}\right]^{e}}\right\}^{-1}$

CAMACHO-MARTINEZ, Humberto Armando, ANTONIOGORDILLO, Diana Montserrat, ROBLES-OCAMPO, José Billerman y SEVILLA-CAMACHO, Perla Jazmín. Deshidratador solar híbrido para el secado de plátano. Revista de Sistemas Experimentales. 2019 


$$
+\frac{\sigma\left(T_{p m}+T_{a}\right)\left(T_{p m}{ }^{2}+T_{a}^{2}\right)}{\left(\varepsilon_{p}+0.00591 N h_{w}\right)^{-1}+\frac{2 N+f-1+0.133 \varepsilon_{p}}{\varepsilon_{g}}-N}
$$

En la ecuación 6, $U_{f o}$ es la conductancia en la parte inferior, donde $k_{a}$ es la conductividad del aislante y $x_{b}$ es la longitud transversal del aislante [2].

$$
U_{f o}=\frac{k_{a}}{x_{b}}=0.22
$$

$U_{l}$ es la conductancia en la parte lateral, las pérdidas de calor a través de los lados del colector se evalúan de manera similar, aplicando la ecuación anterior. Donde $k_{a^{\prime}}$ es la conductividad del aislante lateral, $A_{\text {lateral }}$ es el área y $x_{b}$ es el espesor del aislante por los lados, representada en la ecuación 7 [2].

$U_{l}=\frac{k_{a^{\prime}} A_{\text {lateral }}}{x_{b} A_{c}}=0.24912$

Finalmente, la evaluación del coeficiente total de transferencia de calor para todo el colector está representada por la sumatoria de las tres conductancias indicadas anteriormente; es decir, inferior, superior y lateral [2].

$U_{L}=U_{t}+U_{b}+U_{e}=2.18908$

\section{Diseño}

El diseño del sistema consta de tres componentes principales, los cuales son; una cámara de secado, un colector solar y la unión entre estos, estas tres secciones fueron diseñadas cada una con un propósito en específico.

En la siguiente Fig. 3 se observa la cámara de secado, se diseñó con el espacio suficiente para la colocación de 4 bandejas para la materia a secar, las cuales suman una capacidad total de $15 \mathrm{~kg}$. Incluye una salida de aire la cual esta destinada para la colocación del ventilador, a la vez que deja espacio disponible para poder tener una mayor área para la convección natural. La cual se encuentra rellena de aislante térmico para evitar pérdidas.

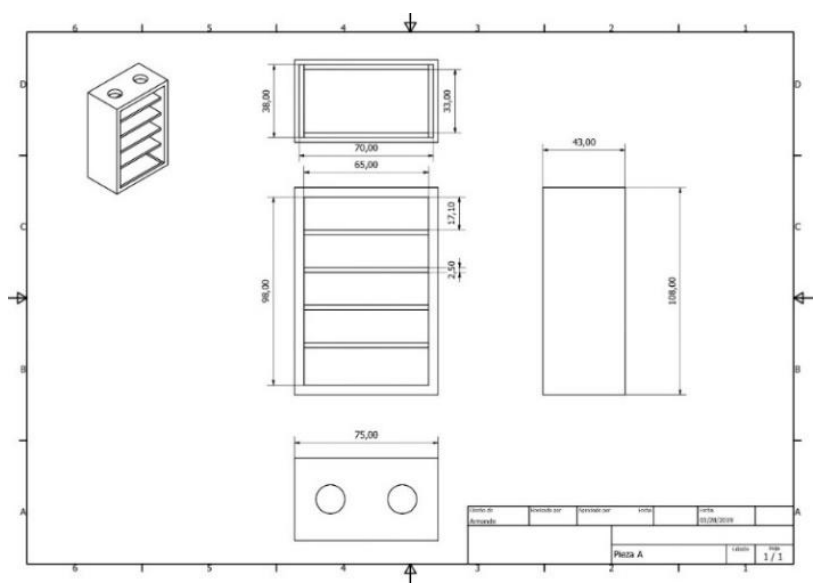

Figura 3 Cámara de secado

El diseño del colector se encuentra en la Fig. 4, el cual cuenta con área superficial de 0.89 $\mathrm{m}^{2}$, a su vez en esta misma estructura se depositan las rocas que actúan como almacenamiento térmico. En su diseño también se incluye una entrada de aire, en la cual se encuentra ubicado el ventilador de entrada para la convección forzada. Su principal función es la de captar la radiación solar, para poder aprovecharla y aumentar la temperatura del sistema.

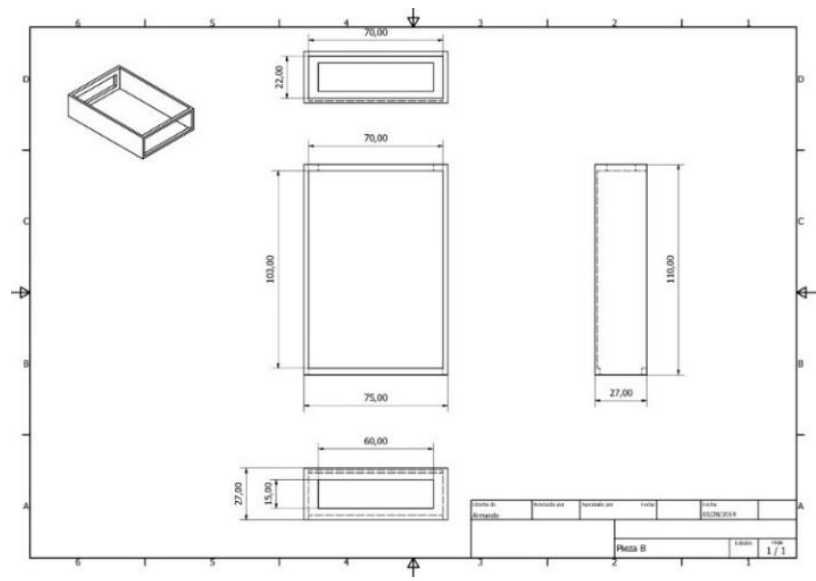

Figura 4 Colector solar plano

En la Fig. 5 se muestra el plano de la unión entre el colector y la cámara de secado. El cual se encontraba recubierto por aislante térmico, con la intención de disminuir en la medida de lo posible las pérdidas de calor en el sistema. 


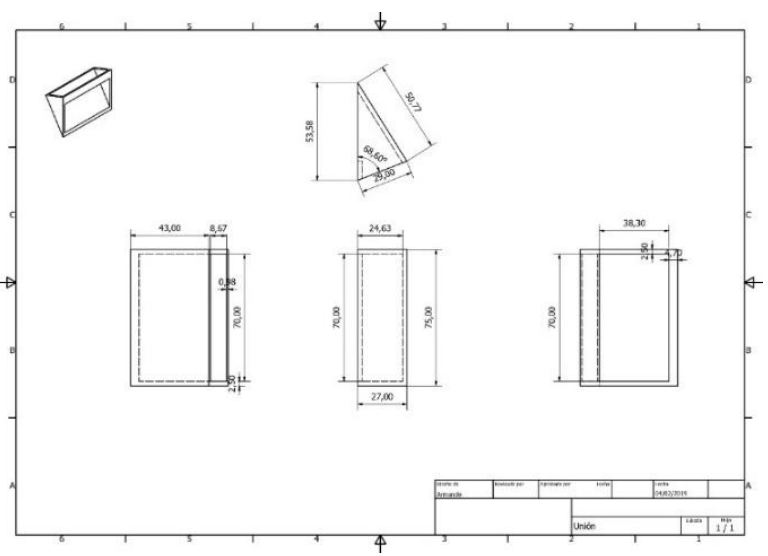

Figura 5 Unión de la cámara de secado con el colector solar

En la Fig. 6 se observa el deshidratador puesto en marcha y en operación, el cual cuenta con una capacidad de $15 \mathrm{~kg}$ con producto a deshidratar.

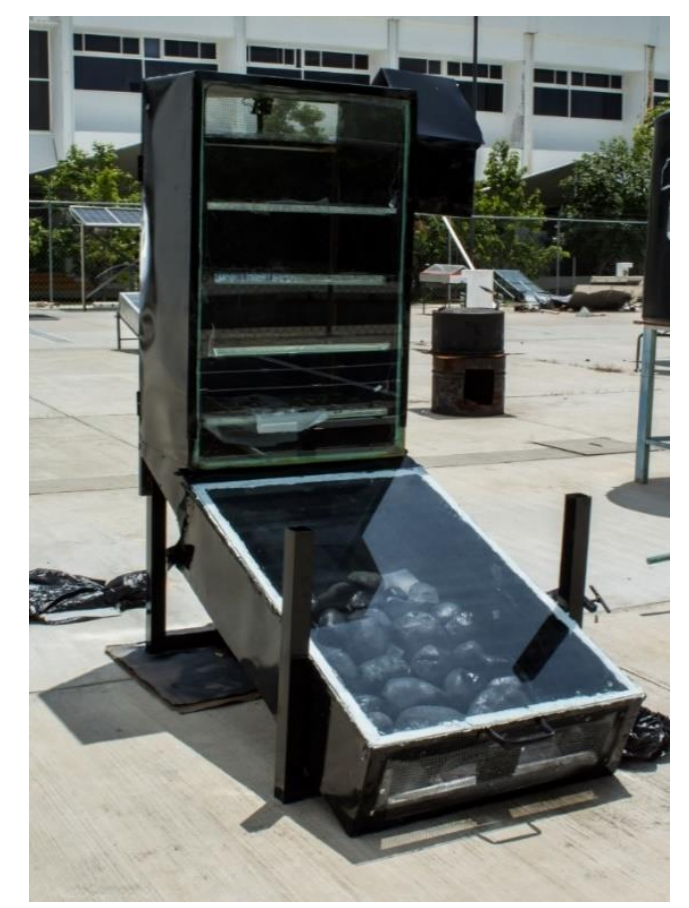

Figura 6 Deshidratador solar híbrido

\section{Resultados y discusión}

Para la caracterización del sistema se llevaron a cabo varias pruebas, tanto teóricas o prácticas. Las pruebas teóricas fueron realizadas en base a el diseño final, teniendo en cuenta las propiedades térmicas y ópticas de los materiales utilizados, como el vidrio, el aislante térmico o la lámina galvanizada. Todo esto con el fin de poder utilizar un modelo matemático para poder predecir el comportamiento ideal del deshidratador.
En la Fig. 7 se muestra una simulación del deshidratador realizada en el software Energy2D. La cual tiene en cuenta las propiedades físicas de los materiales, las dimensiones reales del prototipo, así como también son incluidas las condiciones climatológicas y atmosféricas de la localidad en la cual se llevaron a cabo las pruebas.

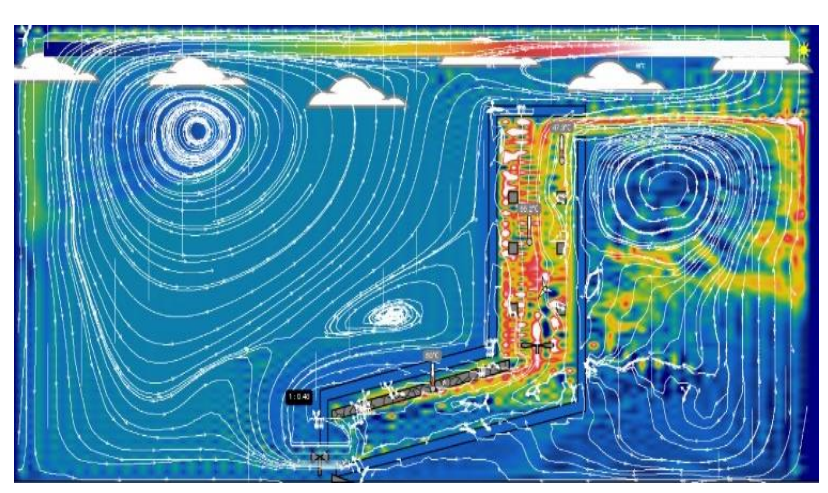

Figura 7 Simulación del funcionamiento del deshidratador

De forma simultánea se realizaron mediciones de las temperaturas presentadas dentro del deshidratador, en la cámara de deshidratado como en el área del colector. Eso con el fin de poder observar el comportamiento de la temperatura en las diferentes secciones del prototipo a lo largo de un periodo de 9 horas. Las pruebas realizadas al deshidratador se realizaron teniendo en su interior $10 \mathrm{~kg}$ de plátano.

En la Fig. 8 se presenta un gráfico en el cual se encuentra el comportamiento de la temperatura atreves del periodo de tiempo de prueba. En este se puede apreciar el efecto que tiene la implementación de un sistema de almacenamiento al pasar las horas pico de radiación.

Así como también es la variación de las temperaturas en las dos secciones caracterizadas del prototipo. 


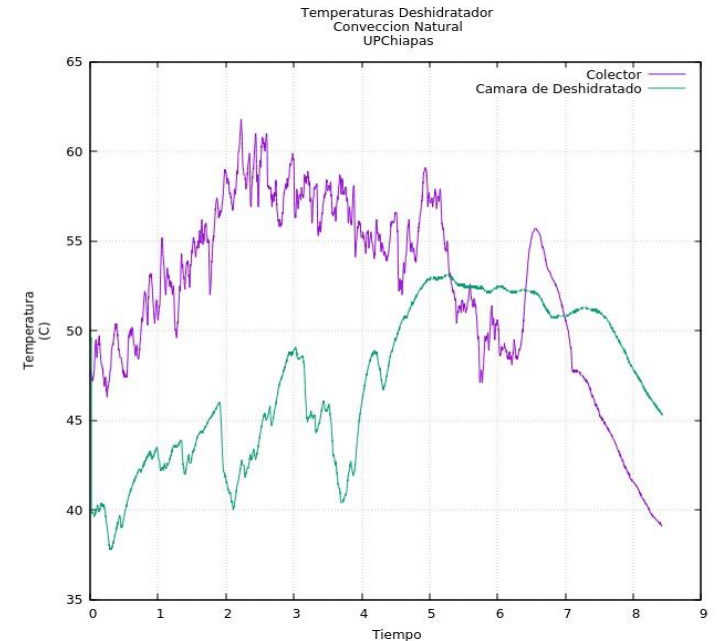

Figura 8 Variación de temperatura en el deshidratador y cámara de deshidratado respecto al tiempo

\section{Conclusión}

El secado de frutas permite la disminución del desperdicio en los alimentos. En el caso del plátano, cuando es deshidratado estando en una etapa de madurez, se obtienen un producto de buena apariencia. Las características de madurez, así como el pretratamiento en la preparación del producto antes del proceso son factores importantes que contribuirán en la calidad del producto final. La temperatura es un factor que afecta directamente a la calidad del plátano deshidratado, dándole apariencia poco agradable.

La simulación térmica nos arroja datos los cuales no son alcanzados por las mediciones experimentales realizadas en las pruebas, sin embargo, las mediciones experimentales presentan una tendencia al comportamiento predicho por la simulación, lo que indica que los principios físicos con los que fue diseñado son apropiados para el correcto funcionamiento.

Dejando además un margen de mejora en la manufactura del prototipo, así como en la calidad de los materiales, acciones que de ser tomadas pueden mejor en gran manera la eficiencia del sistema y por ende las temperaturas, así como el tiempo que temperaturas altas pueden ser mantenidas por el sistema. En cuanto al sistema de almacenamiento pasivo de temperatura, este mostro ser de utilidad en la Fig. 8, esto se concluye del aumento de temperatura que se presenta en la cámara de deshidratado que ocurre de manera simultánea a la caída de la radiación solar, ya que la energía almacena es liberada en los momentos que la radiación solar decae.
De esta forma extendiendo alrededor de 2 horas el tiempo de secado después de las horas d mayor radiación. Las pruebas llevadas a cabo de forma experimental presentaron picos de hasta $65{ }^{\circ} \mathrm{C}$ lo que supera las temperaturas pico de la simulación, esto se atribuye a la inyección de calor de la resistencia eléctrica, la cual permite al sistema llegar a temperaturas las cuales usualmente no alcanzaría.

El conjunto de los sistemas de ganancia de calor (almacenamiento pasivo y resistencia eléctrica) y el sistema de medición y control otorgan al sistema la capacidad de poder alcanzar las temperaturas necesarias para poder llevar a cabo de forma adecuada el proceso de deshidratado de la mayoría de los frutos, así como el poder tener un mayor tiempo útil en el día, extendiendo hasta 2 horas el tiempo de aprovechamiento de la energía solar.

\section{Referencias}

[1]lodoaldo Sivipaucar, H. C. (s.f.). Cálculo y construcción de un secador solar por conveccion natural

[2]Luis Eduardo García, M. F. (2012). Diseño y construcción de un deshidratador solar de frutos.

[3]Venalonzo, R. A. (Octubre de 2017). Diseño y construcción de un secador solar para frutas. Obtenido de https://www.researchgate.net/publication/32 3759795_diseno_y_construccion_de_un_sec ador_solar_para_frutas

[4]Brenan, J.G., (1980). Las operaciones de la ingeniería de los alimentos, vol. 2, 200.

[5]Molano C., Linda G., (2011). Evaluación Nutricional de la Uvilla ( Physalis peruviana L.) Deshidratada, a tres Temperaturas Mediante un Deshidratador de Bandejas, vol. 31.

[6]Oliver Ramírez, A. (2009). Integración de materiales de cambio de fase en placas de yeso reforzadas con fibras de polipropileno aplicación a sistemas de refrigeración y calefacción pasivos para almacenamiento de calor latente en edificios (Doctoral dissertation, Arquitectura). 
[7]Riofrío, A., Vaca, D., Orozco, D. C. M., \& Martínez, J. (2014). Análisis del consumo energético en procesos de cocción eficiente para el sector residencial. In Memorias del Congreso latinoamericano de ingeniería mecánica Colim (Vol. 8, pp. 268-273). 